\section{Avian Influenza: Science, Policy and Politics}

\author{
Ian Scoones, editor
}

Earthscan Publications Ltd., London, UK, 2010

IBSN: 978-1-84971-095-4

(hardcover)

IBSN: 978-1-84971-096-1

(paperback)

Pages: 261; Price: US \$39.95

Pandemic (H1N1) 2009 reiterated the lesson that emerging diseases will continue to challenge us. This pandemic once again brought emerging infectious diseases to the world's attention. Pandemics have occurred for centuries and will continue to occur. The question remains as to what we can learn from the past as we address future pandemics and infectious disease crises. Globalization is inexorable, and global spread of disease occurs faster than ever. Shifting wealth and geopolitical power and balance must affect our response as the world's population becomes increasingly urbanized. As populations change, there will be higher expectations for health, higher anxiety in populations as their health is threatened, and a growing distrust in governmental response.

Severe acute respiratory syndrome and avian influenza (H5N1) confirmed that globalization has altered the developing world's relationship with the industrialized world. Indonesia, the epicenter of the avian influenza (H5N1) epidemic, illustrates the geopolitical debates about equity, public goods, and global responsibilities, as demonstrated in the controversy surrounding virus sharing.

Avian Influenza: Science, Policy and Politics offers insight from the avian influenza (H5N1) response into the wider dilemmas regarding animal health, production and trade, public health, emergency response, and longterm development. Disease threats have transnational implications oper- ating in a complex multilateral system. A one-size-fits-all response is not the answer. Although we think globally, our actions are local: livelihoods affected are local, ecology impacted is local, and disease dynamics are local. Surveillance and disease response systems must be congruent with local social, political, and cultural realities.

The authors provide an in-depth analysis of the political and economic structure of Cambodia, Vietnam, Indonesia, and Thailand and illustrate the many actors and networks involved in policy and response to avian influenza (H5N1) and to intersections of science and politics. These examples uncover key insights into how policy was formulated, and it is this often disguised arena, in which some of the most important indicators of future actions and options are found, that can open up the experience to a wider, more nuanced debate. The authors note, "taking seriously the politics of policy, and not just focusing on the technical or economic dimensions, is vital for a more complete understanding of what works and what doesn't as well as ensuring that the trade-offs between different disease control pathways are made clear.”

This book provides a well-documented approach to laying out issues involved in features of an effective, equitable, accountable, and resilient response infrastructure at international, national, and local levels for influenza and other emerging infectious diseases. The ultimate challenge will be to interpret and implement such recommendations locally, regionally, and globally. The chapters are well written and complete with multiple examples of science, policy, politics, actors, and networks involved in such efforts. This book would interest public health practitioners, those involved in policy and emergency response, and anyone interested in intricacies of policy development. It is essential reading for those involved in infectious disease detection and control and is highly recommended for epidemiologists; human and veterinary medicine practitioners; experts in international health and policy formulation; and persons involved in nongovernmental organizations, political science, business, and industry.

\section{Kathleen Gensheimer}

Author affiliation: Sanofi Pasteur, Cambridge, Massachusetts, USA

\section{DOI: 10.3201/eid1702.101702}

Address for correspondence: Kathleen Gensheimer, Scientific and Medical Affairs, Sanofi Pasteur, 38 Sydney St, Cambridge, MA 02139, USA; email: kathleen.gensheimer@ sanofipasteur.com

\section{Bacterial Population Genetics in Infectious Disease}

D. Ashley Robinson, Edward J. Feil, and Daniel Falush, editors

John Wiley and Sons, Inc., Hoboken, New Jersey, USA, 2010 ISBN: 978-0-470-42474-2

Pages: 420; Price: US $\$ 130.00$

Bacterial Population Genetics in Infectious Disease addresses a set of issues that are extremely provocative in the current climate in which progress is rapidly changing the microbiological landscape. From this perspective, the volume appears at a key moment because of 2 major challenges that have arisen since the beginning of the genomic era: 1) defining what constitutes a species and how to determine this, and 2) determining the structure of the population of the most common bacterial pathogens.

The book opens with a review of the concepts and methods that make it possible to analyze bacterial popu- 
lations genetically. This presentation immediately brings to the forefront the question of how to define what constitutes a bacterial species (if there is conceptually even such a thing) and the variable evolutionary nature of bacteria. The differences among complexes within species, species, and clones are shown with clear diagrams useful for teaching. In addition, the text also evaluates the lateral transfer of genes, one of the elements that constitute the bacterial genome repertoire, including its effects on the attempts at classification of bacteria. The evolutionary demography of bacterial populations is then examined. These concepts are essential for comprehending and teaching modern microbiology.

In addition, the text describes techniques for evaluating bacterial diversity, such as the search for singlenucleotide polymorphisms or repeats by using sequences encoding proteins or not. These techniques can play a key part in the identification of the clones.
The second part of the book is specifically relevant to clinical microbiology, particularly to emerging bacterial pathogens. The genetic populations of Bacillus anthracis, Campylobacter spp., Enterococcus spp., Borrelia burdorferi, Neisseria meningitidis, Escherichia coli, Salmonella spp., Staphylococcus spp., and Streptococcus spp. are analyzed. The importance of genomic recombinations in microbial evolution is particularly stressed for Streptococcus spp., Neisseria spp., and E. coli, for which the number of recombinations is considered to be higher than that of the number of changes; this conclusion likely alters our perception of the evolution of bacteria. Lastly, a general hypothesis is proposed for Salmonella spp.: that their antigens are selected by passage through specific phagocytic protists from their animal hosts with which they have cospeciation. This assumption is bold and intriguing.

On the whole, this book is of general interest for teachers of microbiol- ogy, who need to explain bacteriology in the genomic age, and it may also help clinical microbiologists choose tools for identifying circulating clones. Finally, it can be useful to specialists in the field of emerging bacteria for whom the need to synthesize genomic data is part of their professional routine. I recommend this book most strongly for all of these bacteriology specialists.

\section{Didier Raoult}

Author affiliation: Université de la Méditerranée, Marseille, France

DOI: 10.3201/eid1702.101678

Address for correspondence: Didier Raoult, Unité des Rickettsies, CNRS-IRD UMR 6236198, Université de la Méditerranée, Faculté de Médecine, 27 Blvd Jean Moulin, 13385 Marseille CEDEX 5, France; email: didier. raoult@gmail.com

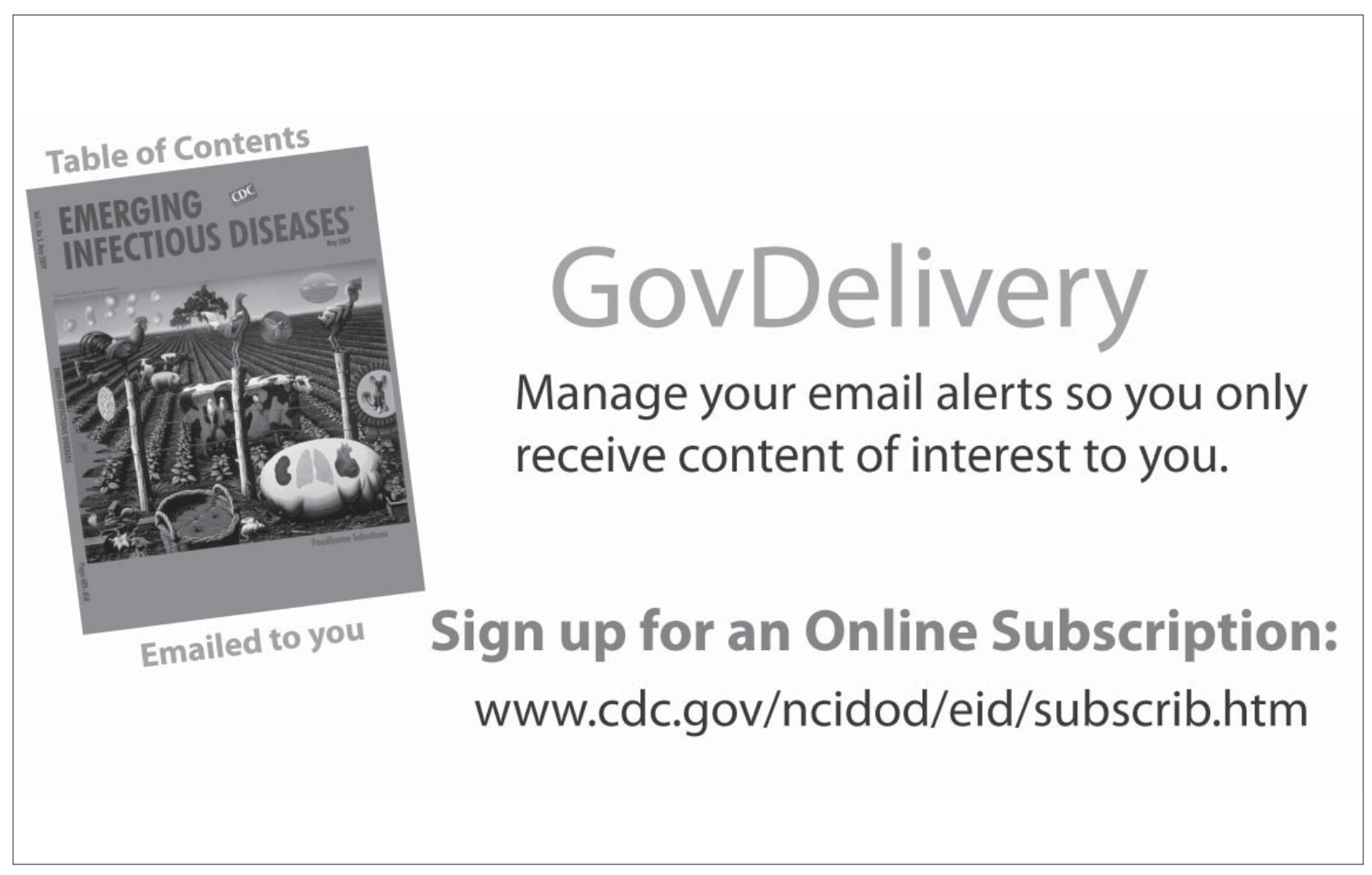

